\title{
NALP12-Associated Hereditary Periodic Fever Syndrome
}

National Cancer Institute

\section{Source}

National Cancer Institute. NALP12-Associated Hereditary Periodic Fever Syndrome. NCI

Thesaurus. Code C119043.

An autoinflammatory disease caused by mutations in the NLRP12 gene. It is

characterized by periodic fevers beginning in the first year of life that are triggered by

cold exposure. Episodes occur more than once per month. 